\title{
Exacerbation of Ulcerative Colitis with Tocilizumab: A Report of Two Cases, One with Takayasu Arteritis and the Other with Relapsing Polychondritis
}

\author{
Yusuke Hanioka, Katsushu Shimizu, Keiko Yamagami, Shuhei Yao, Ryota Nakamura, \\ Tomoyuki Nakamura and Hitoshi Goto
}

\begin{abstract}
:
Tocilizumab (TCZ), a biologic that blocks the signal transduction of interleukin-6, has been used for the treatment of various autoimmune diseases. Many of these cases are sometimes complicated by ulcerative colitis (UC). However, the effect of TCZ on UC is unclear. We experienced two cases with concomitant UC that were treated with TCZ, one for Takayasu arteritis (TAK) and the other for relapsing polychondritis (RP). TCZ did not improve UC in either of these cases. TCZ might have adverse effects on the intestinal tract, since interleukin-6 signaling plays an important role in intestinal epithelium maintenance. Treatment with TCZ should therefore be carefully provided in patients complicated with UC.
\end{abstract}

Key words: ulcerative colitis, tocilizumab, Takayasu arteritis, relapsing polychondritis

(Intern Med 60: 1615-1620, 2021)

(DOI: 10.2169/internalmedicine.5215-20)

\section{Introduction}

With the advent of biologics in recent years, the outcomes of autoimmune diseases and inflammatory bowel diseases, such as Takayasu arteritis (TAK), relapsing polychondritis (RP) and ulcerative colitis (UC) have greatly improved. TAK, RP and UC are all inflammatory diseases of unknown etiology, which might involve autoimmune mechanisms. These diseases involve large vessels, cartilage and colorectal mucosa, respectively, as the focus of inflammation, and each can cause serious or fatal complications. Hence, their control is essential. However, there are refractory cases of these conditions, in which remission cannot be induced even with the combination of steroids and immunosuppressive agents. The biologic tocilizumab (TCZ) is a humanized monoclonal antibody against the interleukin-6 (IL-6) receptor, that exerts strong anti-inflammatory effects by blocking the signaling of IL-6, a typical proinflammatory cytokine. TCZ has become one of the mainstays in the treatment of TAK, because it is expected to reduce steroid dose and induce remission in patients who are refractory to previous treatment (1). In RP as well, the effectiveness of various biologics, including TCZ, has been reported (2). Additionally, the status of biologics in the treatment of UC has been well established prior to their use for TAK and RP (3). Clinically, TAK and UC are often seen in the same patient, as is $\mathrm{UC}$ and $\mathrm{RP}(4,5)$. Therefore, there might be several opportunities to use TCZ in patients who also have UC. However, since the biologics usually used as the first line therapy for UC include tumor necrosis factor inhibitors, there are only few reports of cases of UC treated with TCZ. Hence, the safety and efficacy of TCZ for UC is currently unknown. We herein report two cases that were treated with TCZ, one for TAK and the other for RP, both of which were complicated with UC, along with a review of the relevant literature.

\section{Case Reports}

\section{Case 1}

A 17-year-old woman was admitted to our hospital with fever, diarrhea and melena. The blood pressure in her left arm $(74 / 54 \mathrm{mmHg})$ was lower than in the right $(108 / 60$ 

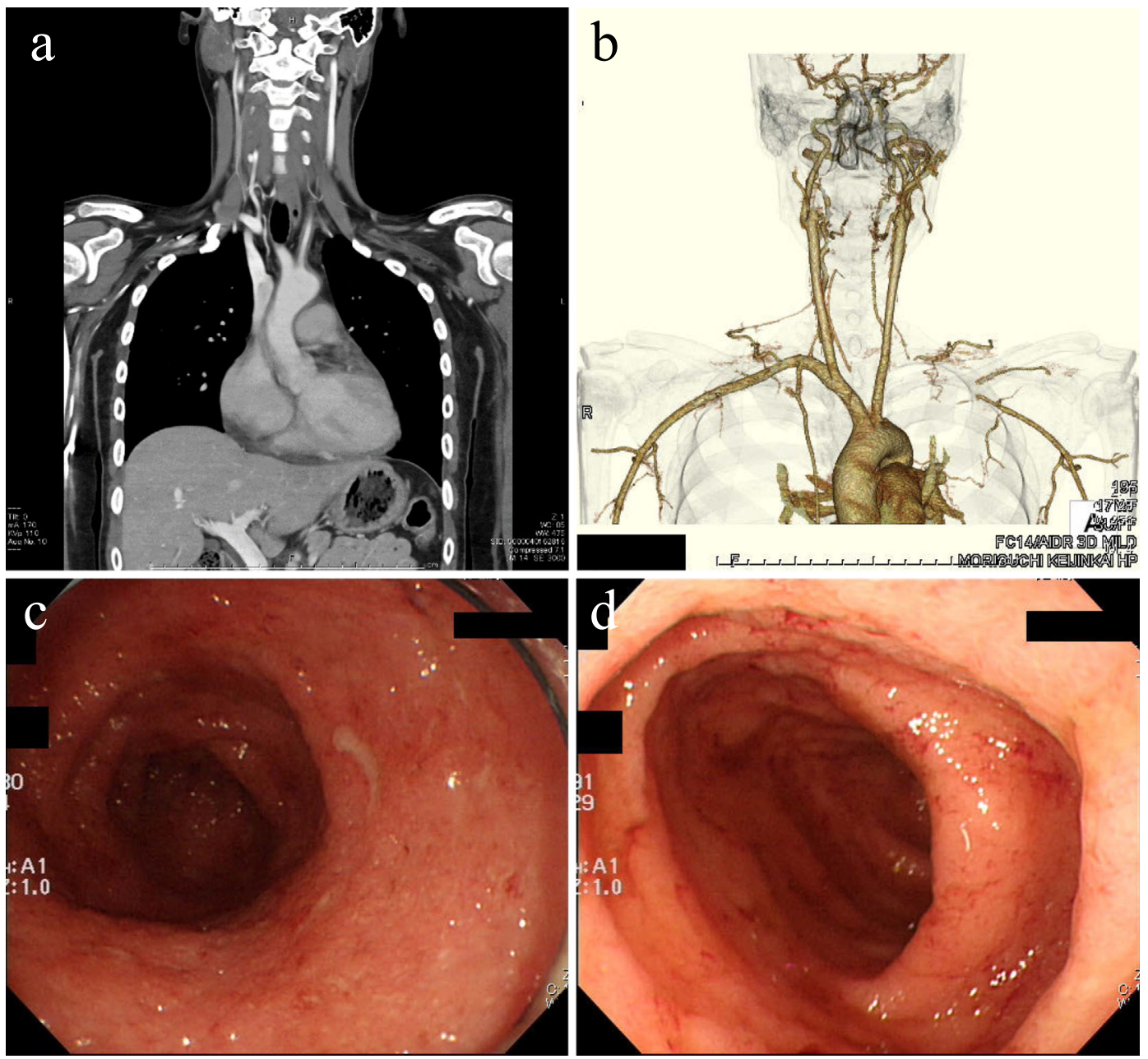

Figure 1. Findings of enhanced CT, CT-angiography and colonoscopy in case 1. Enhanced CT scan showed vessel wall thickening in bilateral common carotid arteries, and the left subclavian artery was completely occluded, as seen on CT-angiography (a, b). Colonoscopy revealed mucosal edema and erosion of the entire colon, leading to ulcerative colitis $(\mathbf{c}, \mathbf{d})$.

$\mathrm{mmHg}$ ), and computed tomography (CT) showed occlusion of the subclavian artery and thickening of the vascular wall in the aortic arch and the common carotid arteries bilaterally. Additionally, colonoscopy revealed mucosal edema and erosion of the entire colon, leading to a diagnosis of TAK and UC (Fig. 1). Her partial Mayo score (pMS), a simplified UC activity measure, was evaluated as 9 at this time. High dose prednisolone (PSL) therapy (1 $\mathrm{mg} / \mathrm{kg} /$ day $)$ and 5aminosalicylate were started, which led to remission and her discharge from the hospital, although the PSL dose could not be reduced to less than $17 \mathrm{mg}$ orally daily due to TAK relapse with steroid reduction, despite combination treatment consisting of steroid therapy with tacrolimus (TAC) and methotrexate (MTX). No diarrhea or hematochezia were observed during this period, the disease activity of UC seemed to have passed at a low level. After about a year and a half, TCZ became available for TAK in Japan. Therefore, on day 502 after her initial visit, we started the intradermal injection of $162 \mathrm{mg}$ TCZ once every two weeks. Since TCZ improved vasculitis, we once again attempted to reduce the steroid dose. However, the UC worsened with reduction of
PSL, although TAK activity remained low. Positron emission tomography (PET) showed little fluorodeoxyglucose (FDG) uptake in the main artery, but with a high accumulation from the descending colon to the rectum; the maximum standardized uptake value (SUVmax) was 9.7 on day 800 after her initial visit. Her pMS was 5 at this time. We continued TCZ therapy after adjusting the steroid dose and immunosuppressive agents for 6 months. As a result, the treatment was maintained for about three months as described below: PSL $10 \mathrm{mg}$ daily, TAC $8 \mathrm{mg}$ daily, and MTX $14 \mathrm{mg}$ weekly. The steroid dosage was not increased at this stage because she did not give her consent. Subsequently, a second PET performed on day 947 after her initial presentation showed no satisfactory effect on the UC. Her pMS remained at 5-6, which was interpreted as no clinical improvement. Hence, we discontinued TCZ and switched to infliximab (IFX) $5 \mathrm{mg} / \mathrm{kg}$, which led to an improvement in her gastrointestinal symptoms without any exacerbation of TAK. The FDG uptake by the colon was also reduced. Her pMS decreased to 1 . The clinical course of this case is shown below (Fig. 2) IFX was administered at two, four and eight weeks 

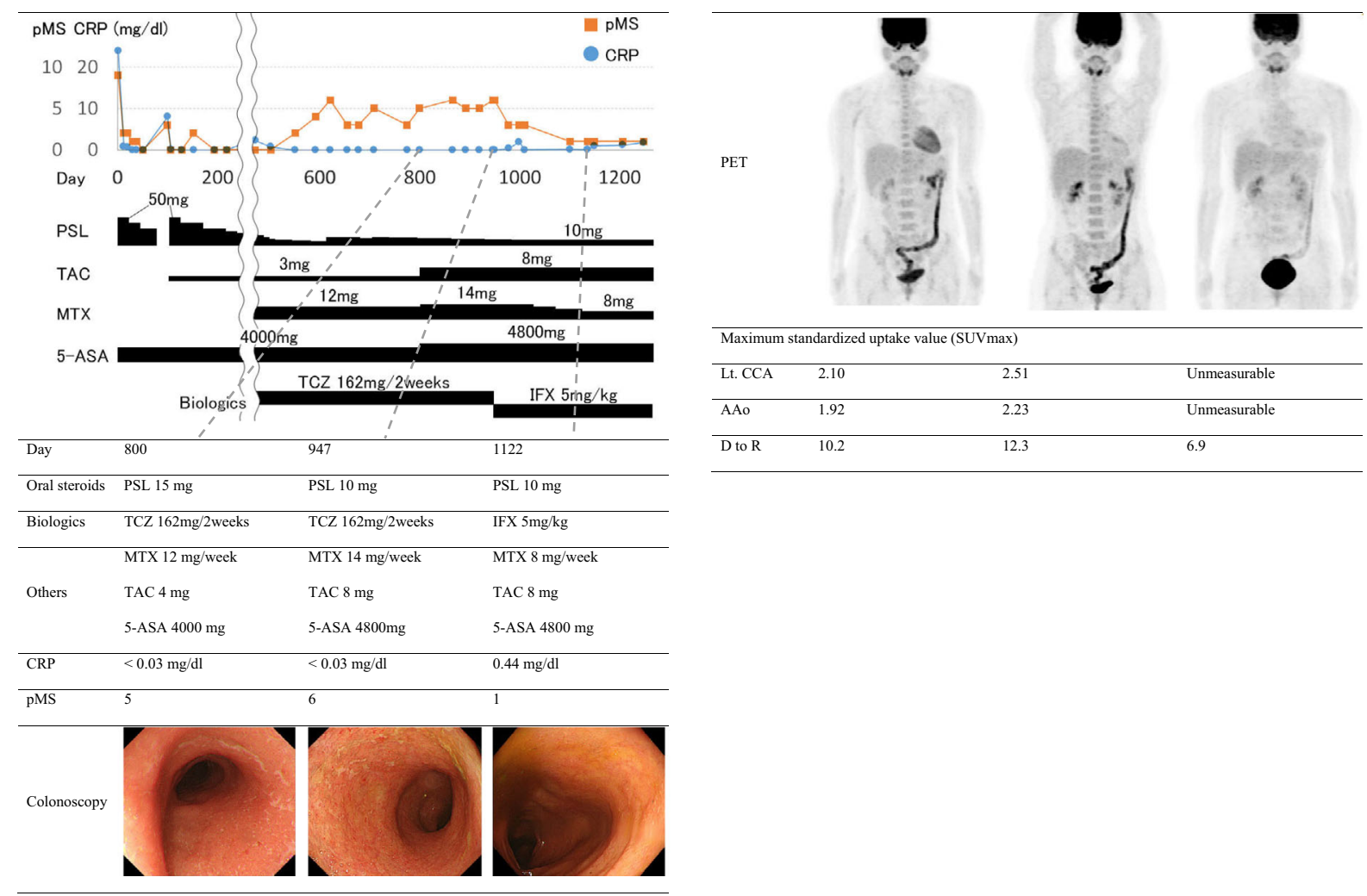

Figure 2. Clinical course of case 1. TCZ was started on day 502 after the initial visit, and PET-CT was performed on days 800, 947 and 1122. Colonoscopy was also performed about one week before or after each PET-CT scan. TCZ improved vasculitis, but was not effective for colitis. TCZ failed to reduce pMS, and PET-CT showed no reduction in SUVmax of the descending colon to rectum. Conversely, IFX improved both clinical symptoms and SUVmax of the descending colon to rectum, without relapse of TAK. 5-ASA: 5-aminosalicylate, AAo: ascending aorta, CRP: C-reactive protein, D to R: descending colon to rectum, IFX: infliximab, Lt. CCA: left common carotid artery, MTX: methotrexate, Others: other immunosuppressive agents, PET: positron emission tomography, pMS: partial Mayo score, TAC: tacrolimus, TCZ: tocilizumab

after the first dose, and then every eight weeks thereafter.

\section{Case 2}

A 72-year-old man was admitted for a fever of unknown origin. Bilateral auricular redness and saddle nose were observed. He also suffered from polyarthralgia and swelling, which seemed to be consistent with rheumatoid arthritis. There was no abdominal pain at this time, but diarrhea was observed. Contrast-enhanced CT did not reveal any significant findings other than multiple diverticula in the sigmoid colon. Auricular cartilage biopsy was performed and RP was diagnosed. High dose PSL $(1.2 \mathrm{mg} / \mathrm{kg})$ led to an immediate improvement in inflammation, and arthralgia and diarrhea also disappeared. However, it relapsed when the PSL dose was reduced to $8 \mathrm{mg}$ daily. Arthralgia also recurred along with the inflammation. Cyclosporine A therapy was added, but it could not be continued due to renal impairment. Since a previous report described the successful treatment of RP with TCZ (2), and our patient's arthritis also met ACR/EULAR criteria of rheumatoid arthritis (6), TCZ was started. As in the previous case, in our case as well, TCZ was effective in treating the chondritis and arthritis. After four weeks of therapy, however, he developed diarrhea, melena and abdominal pain. Colonoscopy showed diffuse erosions and multiple ulcers from the rectum to sigmoid colon. A mucosal biopsy showed non-specific inflammatory findings consistent with UC. No intranuclear injection bodies were observed on Hematoxylin \& Eosin staining, and immunohistochemistry showed no evidence of Cytomegalovirus (CMV) infection (Fig. 3a-d). CMV-DNA was not detected by apolymerase chain reaction testing of biopsy tissue. Stool cultures failed to isolate any significant pathogenic microorganisms, including Clostridium difficile. Glutamate dehydrogenase antigen and toxin $\mathrm{A} / \mathrm{B}$ of Clostridium difficile in the stool were also undetectable. A diagnosis of UC was made, and high dose PSL with 5-aminosalicylate (4,800 mg daily) therapy was started and TCZ was discontinued. However, ten days after commencing treatment, perforation of the sigmoid colon occurred, and total proctocolectomy was performed. The resected specimen showed typical evidence of pancolitis, and UC was definitively diagnosed (Fig. 3e-h). 

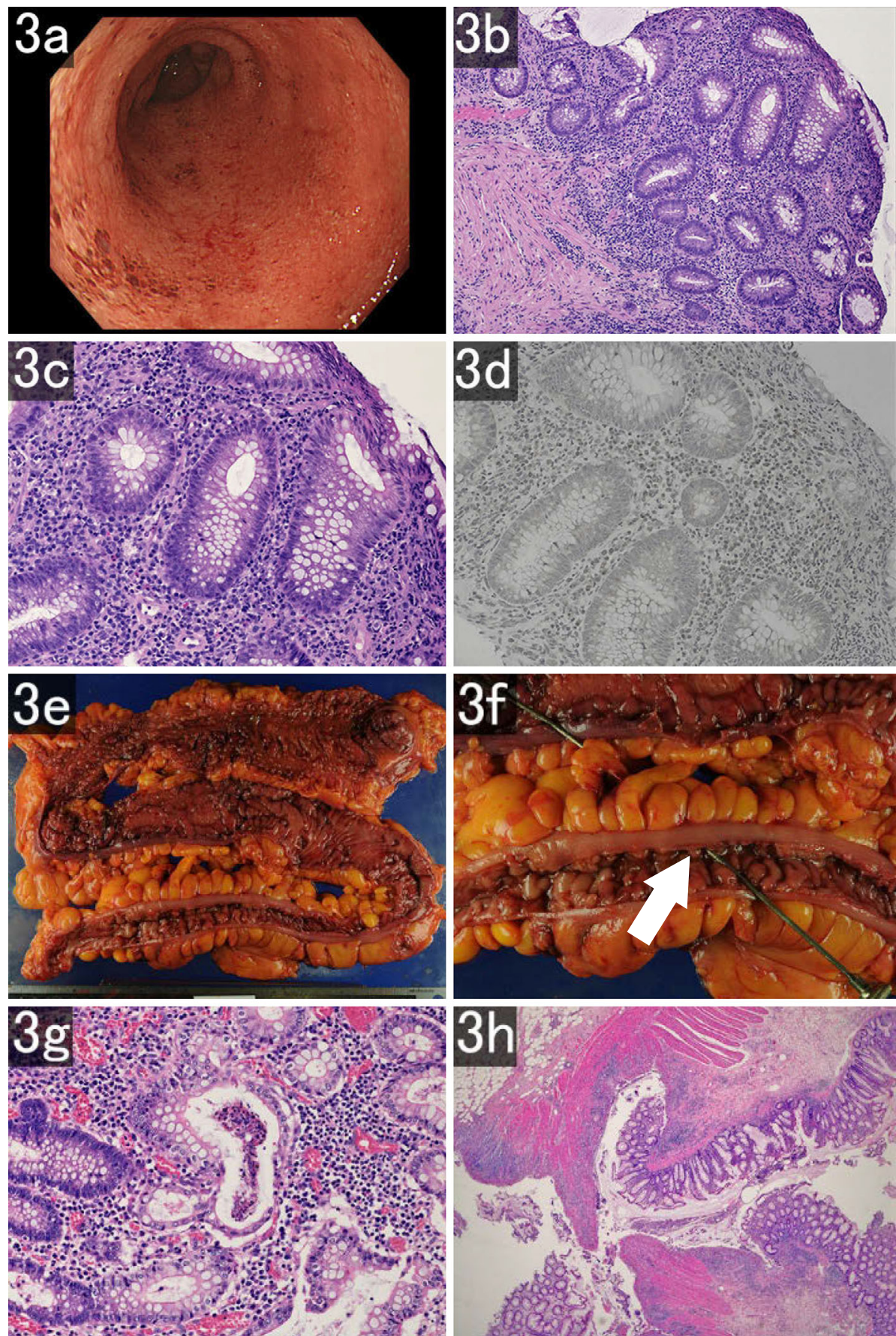

Figure 3. Findings of colonoscopy and the resected specimen in case 2. a: Colonoscopy findings of the sigmoid colon. b: Microscopic findings, low-power field, Hematoxylin and Eosin (H\&E) staining. c: High power field of b. d: Immunohistochemistry study for Cytomegalovirus. Colonoscopy showed diffuse erosions and multiple ulcers from the rectum to sigmoid colon (a). Microscopic findings showed nonspecific inflammation, mainly in the mucosa. There were no nuclear inclusions on $H \& E$ staining and no evidence of CMV infection in immunohistochemistry. e: The resected specimen of the whole colon. $f$ : The site of perforation in the sigmoid colon (white arrow). g: Microscopic findings of the colon mucosa, H\&E staining, high-power field. h: Microscopic findings at the site of perforation, H\&E staining, low-power field. The resected specimen showed perforation of the sigmoid colon and diffuse erosion throughout the entire colon. The residual mucosa showed the findings of pseudopolyps (e, f). Microscopic evaluation showed marked inflammatory cell infiltration and crypt abscesses, consistent with typical UC $(\mathrm{g}, \mathrm{h})$. The site of perforation was infiltrated with inflammatory cells in all layers, but it was not possible to determine whether it was consistent with diverticula. 


\section{Discussion}

$\mathrm{UC}$ is an inflammatory bowel disease of the colonic mucosa that causes multiple erosions and ulcers. Although its etiology remains unclear, it has been suggested to be related to the intestinal microbiome, hereditary factors and malfunction of the immune system. Various indicators are used to measure UC activity, including IL-6. According to previous reports, the IL-6 levels in the serum and colonic mucosa of UC patients increase in association with the disease activity $(7,8)$. Hence, TCZ seems effective in the treatment of UC. However, our cases did not experience any improvement with TCZ. In case 1, UC was improved by changing TCZ to IFX without increasing the dose of steroids and immunosuppressive agents. In case 2 as well, while TCZ was effective for the chondritis, symptoms of UC became apparent and worsened after he started TCZ. Although these results seem to contradict the theory related to the efficacy of TCZ for UC, cases similar to ours have been reported. Atreya et al. reported a case of rheumatoid arthritis with UC that was exacerbated following treatment with TCZ (9). There have also been reports of multiple gastrointestinal ulcer complications other than UC due to $\operatorname{TCZ}(10,11)$. As shown in these cases, TCZ appears to induce some kind of intestinal toxicity, which can be explained by the fact that TCZ inhibits one of the non-inflammatory effects of IL-6, namely, intestinal mucosal protection.

IL-6 is a pleiotropic cytokine that activates not only the inflammatory response, but also hematopoiesis, angiogenesis and other physiological processes. It is also known to be an important cytokine for tissue regeneration and repair (12). Several reports of murine studies showed that IL-6 signaling plays an essential role in proliferation and maintenance of the intestinal epithelium. According to Jin et al., administration of IL-6 causes intestinal epithelial proliferation that increases intestinal volume and reduces reperfusion injury after ischemia. In contrast, IL-6 deficient mice showed impaired intestinal epithelial repair following damage by 5fluorouracil (13). Additionally, Tebbutt et al. showed that IL6 deficient mice had an increased risk of epithelial erosion and mortality compared to wild type mice with dextran sodium sulfate -induced colitis, in a mouse model of UC (14). IL-6 contributes to tissue repair by binding to the IL-6 receptor expressed on cell membranes (mIL-6R) and activating the STAT-3 pathway, via a process called classic signaling. On the other hand, IL-6 also forms a complex with the soluble IL-6 receptor (sIL-6R) that drifts in serum, and induces other signals via glycoprotein 130 (gp130) on the cell membrane, called trans-signaling, which activates the immune system and causes inflammation (15). TCZ exerts anti-inflammatory effects by inhibiting trans-signaling, but since it has a similar affinity for both mIL-6R and sIL-6R, it is expected that intestinal epithelial regeneration is also impaired (16). In our cases, we considered that the mucosal regeneration dysfunction was more problematic than the anti- inflammatory effect of TCZ, leading to a persistence of the mucosal damage and a worsening of inflammation.

Previously, the possibility of adverse effects of TCZ on the intestine has been discussed, and TCZ has been known to carry a greater risk for lower gastrointestinal perforation as compared to non-steroidal anti-inflammatory drugs and other biologics $(17,18)$. Patients with diverticula are considered to be particularly at risk because they are inherently prone to diverticulitis, and there is also a warning about this on the medical package insert (19). This is because of the strong anti-inflammatory effect of TCZ, which can exacerbate infection and mask its symptoms, thereby delaying disease detection. Additionally, it has also been hypothesized that the adipose tissue accumulates locally to cover inflamed diverticula, and this response is prevented by inhibiting IL-6 signaling (20-22). In our opinion, in addition to these factors, delayed wound healing due to classic signaling inhibition might be involved in the adverse effects of TCZ on the intestines. UC is generally less likely to cause perforation than other inflammatory bowel diseases, with an estimated incidence of about 3\%. Approximately $70 \%$ of perforation cases have been reported to be associated with toxic megacolon $(23,24)$, but in our case 2 , there was no evidence of toxic megacolon. Since the perforation point was deeply ulcerated, it was unclear histologically or grossly whether the perforation was consistent with diverticula. It is possible that a diverticulum was perforated, as is the known theory, but if not, perforation is a relatively rare complication of UC, as mentioned above. Our experience suggests that TCZ might be associated with a perforation risk in patients with UC.

Our cases suggest that TCZ has the potential to cause adverse effects on the intestinal mucosa, and it is therefore considered to be a risk factor for UC with TCZ therapy. Certainly, some cases in which TCZ treatment improved UC, as opposed to our cases and those discussed above, have been reported (25-28). The contradictory results seem to be due to the balance between the disadvantages of inhibiting classic-signaling and the benefits of blocking transsignaling. However, patient background factors that might improve or exacerbate the disease remain unknown. As in case 2 , UC is a disease that must be managed with care, because exacerbations of the disease might require emergency surgery or have serious consequences. Although the effect of TCZ on UC has not been reported in sufficient numbers to be conclusive, it should be used with caution given its potential for causing an exacerbation of the disease.

\section{Conclusion}

TCZ therapy should be administered with care in patients complicated with UC, because it can worsen their intestinal condition.

Written informed consent was obtained from both patients for publication of this article.

The authors state that they have no Conflict of Interest (COI). 


\section{References}

1. Nakaoka Y, Isobe M, Takei S, et al. Efficacy and safety of tocilizumab in patients with refractory Takayasu arteritis: results from a randomized, double-blind, placebo-controlled, phase 3 trial in Japan (the TAKT study). Ann Rheum Dis 77: 348-354, 2018.

2. Moulis G, Pugnet G, Costedoat-Chalumeau N, et al. Efficacy and safety of biologics in relapsing polychondritis: a French national multicentre study. Ann Rheum Dis 77: 1172-1178, 2018.

3. Ungaro R, Colombel JF, Lissoos T, Peyrin-Biroulet L. A treatment-to-target update in ulcerative colitis: a systematic review. Am J Gastroenterol 114: 874-883, 2019.

4. Terao C, Matsumura T, Yoshifuji H, et al. Takayasu arteritis and ulcerative colitis: high rate of co-occurrence and genetic overlap. Arthritis Rheumatol 67: 2226-2232, 2015.

5. Cantarini L, Vitale A, Brizi MG, et al. Diagnosis and classification of relapsing polychondritis. J Autoimmun 48-49: 53-59, 2014.

6. Kay J, Upchurch KS. ACR/EULAR 2010 rheumatoid arthritis classification criteria. Rheumatology 51 (Suppl): vi5-vi9, 2012.

7. Neurath MF, Finotto S. IL-6 signaling in autoimmunity, chronic inflammation and inflammation-associated cancer. Cytokine Growth Factor Rev 22: 83-89, 2011.

8. Ishiguro Y. Mucosal proinflammatory cytokine production correlates with endoscopic activity of ulcerative colitis. J Gastroenterol 34: 66-74, 1999.

9. Atreya R, Billmeier U, Rath T, et al. First case report of exacerbated ulcerative colitis after anti-interleukin-6R salvage therapy. World J Gastroenterol 21: 12963-12969, 2015.

10. Hasegawa K. Tocilizumab-induced intestinal injury. Endoscopy 43 : 928, 2011.

11. Iwasa $T$, Nakamura $K$, Ogino $H$, Itaba $S$, Akiho $H$, Okamoto $R$. Multiple ulcers in the small and large intestines occurred during tocilizumab therapy for rheumatoid arthritis. Endoscopy 43: 70-72, 2011.

12. Hashizume M, Ohsugi Y. IL-6 as a target in autoimmune disease and inflammation. Nihon Yakurigaku Zasshi (Folia Pharmaco Jpn) 144: 172-177, 2014 (in Japanese).

13. Jin $X$, Zimmers $T A$, Zhang $Z$, Pierce RH, Koniaris LG. Interleukin-6 is an important in vivo inhibitor of intestinal epithelial cell death in mice. Gut 59: 186-196, 2010.

14. Tebbutt NC, Giraud AS, Inglese M, Jenkins B, Waring P, Clay FJ. Reciprocal regulation of gastrointestinal homeostasis by SHP2 and STAT-mediated trefoil gene activation in gp130 mutant mice. Nat Med 8: 1089-1097, 2002.

15. Rose-John S. IL-6 trans-signaling via the soluble IL-6 receptor: importance for the pro-inflammatory activities of IL-6. Int J Biol Sci 8: 1237-1247, 2012.

16. Mihara M, Katsutani K, Okazaki M, Nakamura A, Kawai S, Sugimoto M. Tocilizumab inhibits signal transduction mediated by both mIL-6R and sIL-6R, but not by the receptors of other members of IL-6 cytokine family. Int Immunopharmacol 5: 1731-1740,
2015.

17. Xie F, Yun H, Bernatsky S, Cutis JR. Brief report: risk of gastrointestinal perforation among rheumatoid arthritis patients receiving tofacitinib, tocilizmab, or other biologics. Arthritis Rheumatol 68: 2612-2617, 2016.

18. Gout T, Ostör AJ, Nisar MK. Lower gastrointestinal perforation in rheumatoid arthritis patients treated with conventional DMARDs or tocilizumab: a systematic literature review. Clin Rheumatol 30: 1471-1474, 2011.

19. Nakajima A. Treatment of rheumaticdisease: current status and future prospective. TopicIII. Biological drugs; 2. IL-6 inhibitor. Nihon Naika Gakkai Zasshi (J Jpn Soc Int Med) 100: 2972-2978, 2011 (in Japanese).

20. Stangfeld A, Richter A, Seigmund B, et al. Risk for lower intestinal perforations in patients with rheumatoid arthritis treated with tocilizumab in comparison to treatment with other biologics or conventional synthetic DMARDs. Ann Rheum Dis 76: 504-510, 2017.

21. Siegmund B, Lehr HA, Fantuzzi G. Leptin: a pivotal mediator of intestinal inflammation in mice. Gastroenterology 122: 2011-2025, 2002.

22. Siegmund B, Sennello JA, Jones-Carson J, et al. Leptin receptor expression on $\mathrm{T}$ lymphocytes modulates chronic intestinal inflammation in mice. Gut 53: 965-972, 2004.

23. Uchino M, Ikeuchi H, Matsuoka $H$, et al. Twenty-eight cases of ulcerative colotis complicating perforative peritonitis. Nippon Daicho Komonbyo Gakkai Zasshi (J Jpn Soc Coloproctol) 61: 298-302, 2008 (in Japanese, Abstract in English).

24. Meyers S, Lerer PK, Feuer EJ, Janowitz HD. Predicting the outcome of corticoid therapy for acute ulcerative colitis. Results of a prospective, randomized, double-blind clinical trial. J Clin Gastroenterol 9: 50-54, 1987.

25. Imamura R, Hayashi K, Sada KE, et al. Hemoptysis originating from the bronchial artery in Takayasu arteritis with ulcerative colitis. Intern Med 58: 293-295, 2019.

26. Szeto MC, Yalçın MD, Khan A, Piotrowicz A. Successful use of Tocilizumab in a patient with coexisting rheumatoid arthritis and ulcerative colitis. Case Reports Immunol 2016: 7562123, 2016.

27. Nishimoto N, Nakahara H, Yoshino-Hoshino N, Mina T. Successful treatment of a patient with Takayasu arteritis using a humanized anti-interkeukin-6 receptor antibody. Arthritis Rheumatol 58: 1197-1200, 2008.

28. Shibata A, Kurasawa T, Okada Y, et al. Successful treatment with tocilizumab monotherapy for Takayasu arteritis developing during infliximab therapy in a patient with ulcerative colitis. Modern Reumatology Case Reports 2: 174-176, 2018.

The Internal Medicine is an Open Access journal distributed under the Creative Commons Attribution-NonCommercial-NoDerivatives 4.0 International License. To view the details of this license, please visit (https://creativecommons.org/licenses/ by-nc-nd/4.0/).

\section{(C) 2021 The Japanese Society of Internal Medicine Intern Med 60: 1615-1620, 2021}

